\title{
NEW SUB-MILLIMETER HETERODYNE OBSERVATIONS OF CO AND HCN IN TITAN'S ATMOSPHERE WITH THE APEX SWEDISH HETERODYNE FACILITY INSTRUMENT ${ }^{\star}$
}

\author{
M. RENGEL* ${ }^{*}$ H. SAGAWA ${ }^{\dagger}$, and P. HARTOGH \\ Max-Planck-Institut für Sonnensystemforschung, \\ Max-Planck-Straße 2, D-37191 Katlenburg-Lindau, Germany \\ *E-mail:rengel@mps.mpg.de \\ www.mps.mpg.de/homes/rengel/
}

\begin{abstract}
The origin of the atmosphere of the largest moon of Saturn, Titan, is poorly understood and its chemistry is rather complicated. Ground-based millimeter/sub-millimeter heterodyne spectroscopy resolves line shapes sufficiently to determine information in Titan's atmospheric composition (on vertical profiles and isotopic ratios). We test the capabilities of the Swedish Heterodyne Facility Instrument (SHFI), Receiver APEX-1, together with the Atacama Pathfinder EXperiment APEX 12-m telescope for Titan's atmospheric observations. In particular we present sub-millimeter observations of the $\mathrm{CO}(2-$ 1) and HCN(3-2) lines of the Titan stratosphere with APEX, and with SHFI taken during the Science Verification (SV) instrument phase on March and June 2008. With the help of appropriate radiative transfer calculations we investigate the possibility to constrain the chemical concentrations and optimize the performance of the APEX-1 instrument for inferring vertical profiles of molecular components of the atmosphere of Titan.

This study attempts to contribute to constrain radiative transfer and retrieval algorithms for planetary atmospheres, and to give hints to the current and future ground and space-based data acquisition leading to a more thorough understanding of the chemical composition of Titan.
\end{abstract}

Keywords: APEX-1; abundance; atmosphere; Herschel; Submillimeter; composition; Titan; HssO; Water and Related Chemistry, Retrieval; Heterodyne Spectroscopy.

\footnotetext{
^ Based on observations collected at the European Southern Observatory, Chile, corresponding to the observing proposal number 081.F-9812(A).

${ }^{\dagger}$ Currently at the National Institute of Information and Communications Technology, Japan
} 


\section{Introduction}

Titan's atmosphere exhibits a complex photochemistry. The origin of carbon monoxide (CO) is not well understood (whether photochemical or primordial). Hydrogen Cyanide (HCN), the most abundant nitrile in Titan, is a key intermediate in production of more complex hydrocarbons and organic molecules. HCN in the Titan atmosphere has been discovered by the infrared observations of Voyager $1^{1}$, and CO has been detected by the ground-based near-infrared observations ${ }^{2}$. Following these detections, the molecular concentrations of $\mathrm{CO}$ and $\mathrm{HCN}$ in Titan's atmosphere have been determined from infrared, millimeter, and sub-millimeter observations as well as from modeling (see Tables 2 and 3 in section 4 for details). CO seems to be uniformly mixed in Titan's atmosphere up to high altitudes. HCN abundances, however, display a steeper profile with ambiguous enrichment values (Table 3). Already several observational data confirm that Titan's atmospheric composition is indeed seasonally ${ }^{3,4}$, and spatially dependent ${ }^{5}$. Full behavior of the seasonal characteristics of the spatial distribution is not constrained yet due to the limitation in the temporal coverage of the previous observations, and to the use of different instruments. New submillimeter observations are required not only to provide new abundance constraints and shed more light on the rate of these seasonal variations, for example, but for support, complement and cross-calibration the ESA's Herschel Space Observatory mission. One of the goals of the Key Program of Herschel, entitled Water and Related Chemistry in the Solar System ${ }^{6}$, is to understand water inventory in the Titan's atmosphere as well as distributions of other hydrocarbons and nitriles. During the guaranteed time, line surveys on Titan at the frequency range of 500 and $5000 \mathrm{GHz}$ are going to be carried out using two low-to-medium resolution spectrometers (Photodetector Array Camera and Spectrometer (PACS) and Spectral and Photometric Imaging Receiver (SPIRE)). Our model calculations of the synthetic spectra of Titan expected to be observed with Herschel show that several CO and HCN lines will be detected with PACS and SPIRE? Because of their low-to-medium spectral resolutions, SPIRE and PACS are not capable of measuring the shapes of $\mathrm{CO}$ and $\mathrm{HCN}$ lines, which prevents us from determining precisely the vertical profile of $\mathrm{CO}$ and $\mathrm{HCN}$ mixing ratios. Therefore, high spectral resolution observations of $\mathrm{CO}$ and $\mathrm{HCN}$ with ground-based sub-millimeter telescopes would significantly improve the accuracy of retrieving the $\mathrm{CO}$ and HCN profiles.

The Atacama Pathfinder EXperiment (APEX) 12-m telescope is operational since $2005^{8}$, and has been already used to the planetary science 
(monitoring mesospheric winds of Venus ${ }^{9}$ ), among numerous galactic and extra-galactic objects. In spring 2008, the new APEX Swedish Heterodyne Facility Instrument (SHFI) has been commissioned on the APEX 12-m telescope $^{10,11}$, which consists of four single-pixel receivers (APEX-1, APEX-2, APEX-3, and APEX-T2) mounted in a single cryostat located in the Nasmyth A cabin of APEX. In this report we present a summary of our observations of $\mathrm{CO}$ and HCN performed with APEX-1 during the Science Verification (SV) phase with the aims to demonstrate the capabilities of APEX and SHFI for Titan's atmospheric observations, and to investigate the possible retrieval of $\mathrm{CO}$ and $\mathrm{HCN}$ abundances. We also examine the possible detection of the millimeter/sub-millimeter rotational transition of $\mathrm{C}_{2} \mathrm{H}_{2}$ (already observed in the mid-infrared spectra by the Composite Infrared Spectrometer, CIRS, on Cassini ${ }^{12}$ ), and the search for one new compound, $\mathrm{HC}_{5} \mathrm{~N}(83-82)$ which the possible presence has been suggested by experimental results simulating Titan's atmosphere ${ }^{13-15}$.

The observations are described in detail in section 2 while the radiative transfer modeling and the data analysis are discussed in sections 3 and 4 , respectively. A brief discussion is given in section 5 and a summary in section 6 .

\section{Observations}

Titan's spectra were acquired with the APEX 12-m telescope located on Chajnantor (Chile) during 21 March, and 17-18, 23, and 27 June 2008 during SV. We used the APEX-1 receiver, operating at 211-270 GHz, which employs a superconductor-insulator-superconductor (SIS) mixer and behaves as a single sideband receiver (SSB). For the backend, we used the Fast Fourier Transform Spectrometer (FFTS) with a channel separation of $122 \mathrm{kHz}$ and a bandwidth of $1 \mathrm{GHz}$. Table 1 summarizes the measured transitions as well as the observing days and the precipitable water vapour (PWV). Titan was observed near the western or eastern elongations at separation angles from Saturn greater than 120". Pointing and focusing of the telescope were regularly checked scanning across Saturn in azimuth and in elevation (APEX has a pointing accuracy of $2^{\prime \prime}$ r.m.s. over sky). The apparent diameter of Titan was around $0.8^{\prime \prime}$.

Because a bug in the low-level Python ephemerides software not previously detected, Titan was not tracked properly at the beginning of our observations on 21 March 2008. The bug was successfully removed by the APEX staff. These observations in particular have helped to improve the control software. Accounting for possible frequency errors in the line center 
Table 1. Species observed on Titan

\begin{tabular}{lccc}
\hline Species & Frequency $(\mathrm{GHz})$ & Date 2008 & PWV $(\mathrm{mm})$ \\
\hline $\mathrm{HCN}(3-2)$ & 265.886 & 21 March & \\
$\mathrm{HC}_{5} \mathrm{~N}(83-82)$ & 220.937 & $17-18$ June & $3.5-4.0$ \\
$\mathrm{CO}(2-1)$ & 230.538 & $23 \mathrm{June}$ & 0.5 \\
$\mathrm{HCN}(3-2)$ & 265.886 & 23 June & 0.5 \\
$\mathrm{C}_{2} \mathrm{H}_{2} \mathrm{~J}=47 \nu 5=1 \rightarrow \mathrm{J}=46 \nu 4=1$ & 254.521 & 27 June & 0.2 \\
\hline
\end{tabular}

(of a few $\mathrm{MHz}$ around the respective line centers), and considering the short integration time, we could not detect any evident signature of $\mathrm{HC}_{5} \mathrm{~N}(83-82)$ and $\mathrm{C}_{2} \mathrm{H}_{2}$ in the recorded spectra averages of integration times of 36 and 91 min. (r.m.s. of 0.035 and $0.03 \mathrm{~K}$ ), respectively. Lower r.m.s. noise levels of around $0.01 \mathrm{~K}$ (which could be achieved with integration times of around 8 and $13 \mathrm{~h}$, respectively) would have facilitated the possible detections of these species. Therefore we concentrate here on the $\mathrm{CO}(2-1)$ and $\mathrm{HCN}(3-2)$ observations. Because of the high oversubscription of observing proposals and the uncertainties about the tracking at the beginning of the campaign, the initially proposed integration time of around 2 hours for $\mathrm{CO}(2-1)$ and 2.65 hours for $\mathrm{HCN}(3-2)$, which corresponds to a r.m.s noise level of $0.017 \mathrm{~K}$ and $0.025 \mathrm{~K}$ respectively, was not available. Instead, $\mathrm{CO}(2-1)$ and $\mathrm{HCN}(3-2)$ were integrated for around 13 and $19 \mathrm{~min}$, respectively. The initial reduction of each spectrum was performed using the CLASS software package of the Grenoble Astrophysics Group ${ }^{\mathrm{a}}$.

Fig. 1 presents the recorded Titan's spectra of the $\mathrm{CO}(2-1)$ and $\mathrm{HCN}(3-$ 2 ) lines. The resolution has been downgraded to about $4 \mathrm{MHz}$ in order to increase the signal-to-noise ratio.

The conversion from the observed antenna temperature $T_{a}^{*}$ to the total flux density from the Titan's atmosphere $S_{\nu, t o t}[\mathrm{Jy}]$ is performed by $S_{\nu, t o t}=$ $24.4 \times T_{a}^{*} \cdot \eta_{f} / \eta_{a}$, where $\eta_{f}$ and $\eta_{a}$ are the forward and aperture efficiencies, respectively. In this study, we adopt the values considering the efficiencies listed on the APEX web site ${ }^{\mathrm{b}}$ and of the instrument paper ${ }^{8}: \eta_{a}$ are 39.9 and 40.2 for 230 and $265 \mathrm{GHz}$, respectively, and $\eta_{f}$ is 0.95 for both frequencies. These values are expected to have a $10 \%$ uncertainty.

By fitting the synthetic spectra to the observed one, we intend to infer the abundance of $\mathrm{CO}$ and $\mathrm{HCN}$ in Titan's stratosphere. A precise comparison of the observations with synthetic spectra must cope with two limita-

a URL:http://www.iram.fr/IRAMFR/GILDAS

bURL:http://www.apex-telescope.org/telescope/efficiency/ 

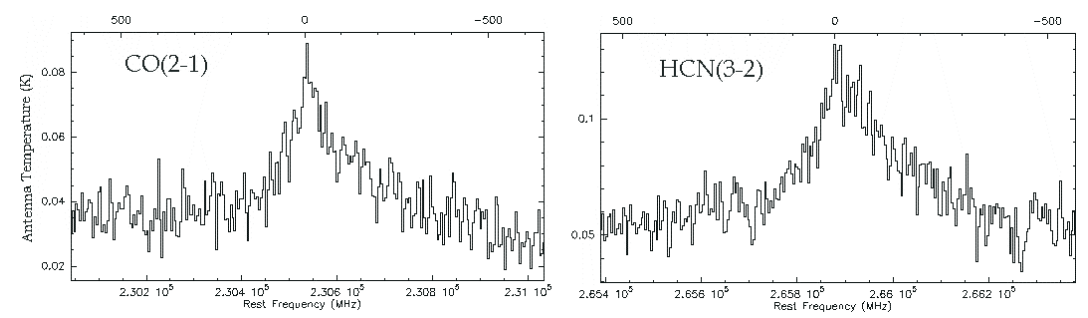

Fig. 1. Titan's spectra observed with the APEX 12-m Telescope on 23 June 2008. Left: $\mathrm{CO}(2-1)$ line rebinned over 32 resolution elements (4 MHz). Right: $\mathrm{HCN}(3-2)$ line rebinned over 32 resolution elements $(4 \mathrm{MHz})$.

tions: (1) the line-to-continuum ratios of these $\mathrm{CO}$ and $\mathrm{HCN}$ spectra were not able to be confirmed from the observed data. $1 \mathrm{GHz}$ bandwidth is too narrow for constraining the continuum level. We have examined the possibility of using the emission at the frequency of $\pm 500 \mathrm{MHz}$ offset from the line center as the pseudo-continuum. We found, however, that the intensities of the $\mathrm{CO}(2-1)$ line at the $\pm 500 \mathrm{MHz}$ significantly depend on the $\mathrm{CO}$ abundance at a certain range of altitude, which also affects the intensity at the line center. Therefore, we conclude that using such fluxes as the pseudocontinuum is not a suitable method for determining the $\mathrm{CO}$ abundance in this study. This is also the case for the HCN (3-2) line. (2) Baseline ripples and uncorrected larger scale baseline characteristics may affect the shape of the observed spectra. One can obtain a spectral line shape symmetric with respect to the line center by folding the spectrum. However, such a spectra folding may introduce an artificial error in the line shape. Therefore we prefer to keep the spectra with their original baseline distortions.

\section{Radiative transfer modeling}

The synthetic spectra are generated by a multi-layered line-by-line radiative transfer model considering the spherical geometry of Titan's atmosphere. It consists of 120 layers that span the $0-600 \mathrm{~km}$ interval with a resolution of $5 \mathrm{~km}$. In general, the atmospheric model for Titan is explained fully in Rengel et al. (2009) ${ }^{7}$. Briefly, Titan's thermal and pressure profiles based on the in-situ measurement by the Huygens probe ${ }^{16}$ are adopted for the opacity calculation of the $\mathrm{CO}$ and $\mathrm{HCN}$ gases at each altitude level. The mixing ratio profiles of $\mathrm{CO}$ and $\mathrm{HCN}$ are set as the parameters to be retrieved in this study. The detail of modeling vertical profiles of each molecule is described later. For $\mathrm{CO}$, the assumed $\mathrm{N}_{2}$-broadening line-width here is 
that of Semmoud-Monnanteuil \& Colmont (1987) ${ }^{17}$, and for HCN, the value corresponding to the same transition at the infrared vibrational-rotational band ${ }^{18}$ as there is no exact measurement for this pure rotational line. The collision induced absorption coefficients of $\mathrm{N}_{2}, \mathrm{CH}_{4}$, and $\mathrm{H}_{2}$ mixtures, which dominate the millimeter and sub-millimeter continuum opacity of Titan's atmosphere, are included by using the formulation of Courtin (1988) ${ }^{19}$ and Borysow and Tang $(1993)^{20}$.

\section{Data analysis}

\subsection{The CO abundance}

Recent observations suggest that CO in Titan is uniformly mixed throughout Titan's atmosphere ${ }^{21-23}$. Such a profile can be supported if its long chemical lifetime in the oxygen-poor Titan atmosphere is considered $\left(\sim 10^{-9}\right.$ years $^{24}$ ). In this study, we retrieve $\mathrm{CO}$ abundance by assuming a vertically constant profile. It should be noted that some of the previous observations have shown the possibility of existence of the discontinuity in the CO mixing ratios between the troposphere and the stratosphere (e.g., $\sim 30 \mathrm{ppm}$ in the troposphere ${ }^{25}$ and $\sim 60 \mathrm{ppm}$ in the stratosphere $\left.{ }^{26}\right)$. However, such discontinuity cannot be constrained by our observations, as the $\mathrm{CO}(2-1)$ measurement reported here does not have any sensitivity to the altitude below $60 \mathrm{~km}$ (Fig. 2 left panel).

A direct comparison of synthetic and observed spectra is made and the best fitting model with the least $\chi^{2}$ is determined. In Fig. 2 right panel, the best-fit $\mathrm{CO}$ abundance shows a solution of $30_{-8}^{+15} \mathrm{ppm}$. Although this solution agrees with the previous stratospheric CO measurements of Hidayat et al. $(1998)^{27}$, Lellouch et. al $(2003)^{25}$, and Baines et al. $(2006)^{22}$ (Table 2), we have to allow a larger error on our result by considering the uncertainty in the flux scaling and the baseline problems. To examine the effect of the uncleaned baseline of the observed spectrum, we performed the fitting analysis by constraining the data only on each side of the wings. Using the red-shift wing $\left(\nu<\nu_{0}\right)$ yielded the best fitting $\mathrm{CO}$ mixing ratio of $26_{-8}^{+13} \mathrm{ppm}$, while using the blue-shift wing $\left(\nu>\nu_{0}\right)$ resulted $36_{-11}^{+15} \mathrm{ppm}$. Taking into account the $10 \%$ uncertainty in the flux calibration, the acceptable range of $\mathrm{CO}$ mixing ratio becomes as $16-51 \mathrm{ppm}$. This wide range covers most of the past works. One way to avoid such a large ambiguity in the data analysis (i.e. to avoid the effect of the uncertainty in the flux calibration) is to calibrate the observed spectrum by scaling its continuum level to that of the forward model calculation. Our forward model calculations 
suggest that the intensity at the frequency offset larger than $\sim 1.5 \mathrm{GHz}$ can be used as the continuum level.
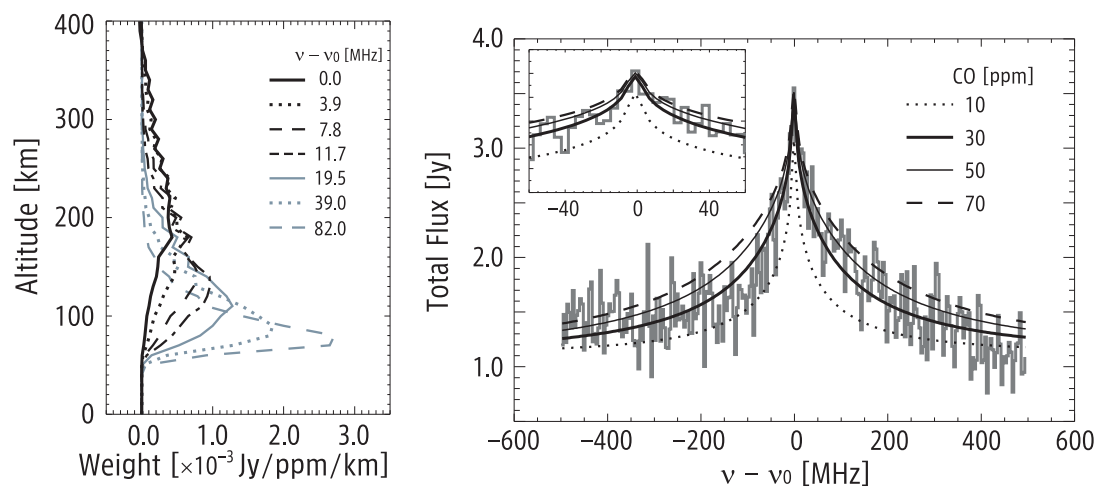

Fig. 2. Left: Weighting functions with respect to $\mathrm{CO}$ mixing ratio for selected frequencies near the $\mathrm{CO}(2-1)$ line center. Note that the finite spectral resolution $(4 \mathrm{MHz})$ of the measurement is taken into account for the calculation of these weighting functions. Right: Observed and synthetic $\mathrm{CO}(2-1)$ spectra at $230.54 \mathrm{GHz}$ (grey spectrum and line ones, respectively). The synthetic spectra are calculated assuming vertically uniform $\mathrm{CO}$ mixing ratios of $10,30,50$, and $70 \mathrm{ppm}$ (from bottom to top) respectively. The small upper panel shows a zoom of the spectrum for a frequency range of $\pm 60 \mathrm{MHz}$.

\subsection{The HCN vertical profile}

We retrieve the mixing ratio profile of HCN by performing forwardand inversion calculations iteratively. For solving the inversion problem, we employ the optimal estimation method (OEM) (e.g., see details in Rodgers $\left.(1976)^{36}\right)$. The Levenberg-Marquardt scheme is used in the iteration. We set the logarithm of HCN mixing ratio as the retrieving parameter. The a priori profile of $\mathrm{HCN}$ mixing ratio, which is required in the OEM approach to regularize the ill-posed inversion problem, is based on the previous ground-based work by Marten et al. $(2002)^{37}$. We assumed the a priori error of 2.3 in the logarithm scale. During the HCN retrieval, the temperature profile is fixed to our nominal temperature profile (the one based on the Huygens measurements).

Fig. 3 shows the best-fit synthetic spectrum (solid line) and the corresponding HCN profile. The averaging kernels of the retrieved HCN mixing ratios indicate that this retrieval has a significant sensitivity at the altitude around $90 \mathrm{~km}$ though we can still retrieve information at the higher 
Table 2. Observations of CO in Titan atmosphere

\begin{tabular}{|c|c|c|c|c|}
\hline Altitude & Mixing ratio $[\mathrm{ppm}]$ & Wavelength $^{*}[\mu \mathrm{m}]$ & Facility/Instrument & Reference \\
\hline Troposphere & $48_{-32}^{+100}$ & 1.57 & KPNO-4m/Fourier Transform interferometer & 2 \\
\hline Stratosphere & $30-180$ & 35314.89 & VLT $-70 \mathrm{~m} / 27$ antennas & 28 \\
\hline Stratosphere & $60 \pm 40$ & 2602.59 & OVRO/2 elements interferometers & 29 \\
\hline Stratosphere & $2_{-1}^{+2}$ & 2602.59 & IRAM-30m/3-mm SIS receiver & 30 \\
\hline Stratosphere & $50 \pm 10$ & 2602.59 & OVRO/6 $10.4 \mathrm{~m}$ diam. antennas & 31 \\
\hline Troposphere & $10_{-5}^{+10}$ & 4.8 & UK IR Telescope/CGS4 spectrograph & 32 \\
\hline Stratosphere & $27 \pm 5$ & $2602.59,1304.34,900.9$ & IRAM-30m/SIS heterodyne receivers & 27 \\
\hline Stratosphere & $52 \pm 6$ & 1304.34 & OVRO $/ 6$ antennas & 33 \\
\hline Troposphere & $32 \pm 10$ & $4.75-4.85$ & VLT 8-m/ISAAC & 25 \\
\hline Stratosphere & $51 \pm 4$ & 869.56 & SMA $/ 5$ and 6 antennas & 21 \\
\hline $153-350 \mathrm{~km}$ & 60 & $4.5-4.85$ & VLT 8-m/ISAAC & 26 \\
\hline Tropo-Stratosphere & $45 \pm 15$ & 4.64 & Cassini/CIRS & 34 \\
\hline Stratosphere & $32 \pm 15$ & 4.64 & Cassini/VIMS & 22 \\
\hline Stratosphere & $47 \pm 8$ & $333.3-166.66$ & Cassini/CIRS & 35 \\
\hline Stratosphere & $30_{-8}^{+15}$ & 1301.29 & APEX/APEX-1 receiver & This article \\
\hline
\end{tabular}

* In spite that each wavelength region is defined by standard specifical units, we use here $\mu \mathrm{m}$ to facilitate the comparisons.

altitudes up to $240 \mathrm{~km}$. Our retrieved $\mathrm{HCN}$ mixing ratios are larger than those of Marten et al (2002) by a factor of $\sim 30-80 \%$ at the altitudes of 100 $200 \mathrm{~km}$. The synthetic spectrum calculated with the a priori HCN profile, i.e., the result of Marten et al. (2002), is also shown for comparison (Fig. 3). A large discrepancy between the observed and the synthetic spectra, and the a priori profile is seen mostly at the wings of the spectrum. This results in the retrieved larger $\mathrm{HCN}$ abundances at the altitudes of $90-150 \mathrm{~km}$. Note that our result showing lower HCN abundance values at around $300 \mathrm{~km}$ is out of the altitude range where our observations are sensitive.

In the above mentioned retrieval, we only took the system temperature of the APEX-1 instrument into account for the statistical error of the measurement. In fact, as we discussed previous in section 4.1, the observed spectrum contains the $10 \%$ uncertainty in the flux calibration. If a $10 \%$ larger scaling factor is applied in the flux calibration, we cannot derive any realistic HCN profiles which can reproduce the observed spectrum. In case of using a $10 \%$ smaller scaling factor, the $\mathrm{HCN}$ abundances for the best-fit spectrum are larger than those of Marten et al. (2002) at the altitude of $100-150 \mathrm{~km}$ (which is attributed by the wings of the spectrum) and then becomes as small as $\sim 30 \%$ of Marten et al. (2002) at higher altitudes of 150-200 km (Fig. 4).

The two retrievals (Figs. 3 and 4) demonstrate the significant impact of 
the flux calibration uncertainty on the retrieved HCN profile. Since we cannot constrain the line-to-continuum ratio of the observed spectrum, we are not able to conclude the HCN abundance at altitude of $150-200 \mathrm{~km}$ which deviates within a wide range from 30 to $150 \%$ of the a priori guess. For the lower altitudes such as $100-150 \mathrm{~km}$, the wing of our observed spectrum requires a higher opacity of $\mathrm{HCN}$ than that of Marten et al. (2002) even if we consider the uncertainty in the flux calibration.
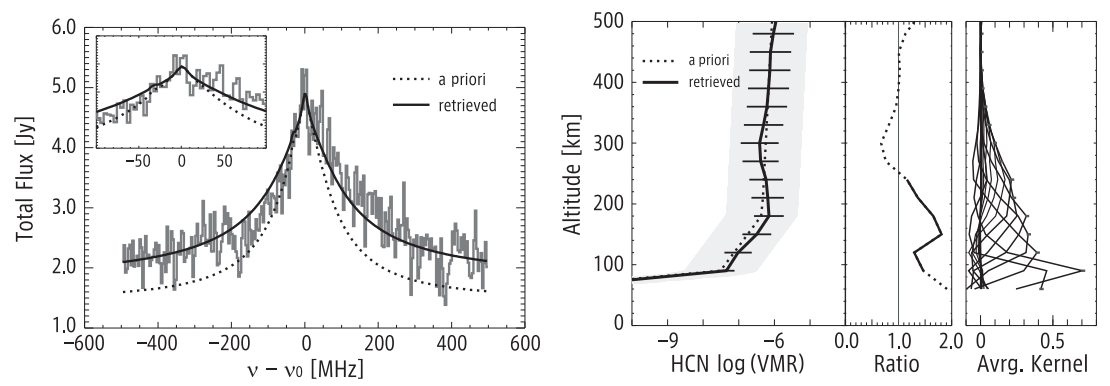

Fig. 3. Left: Observed and synthetic $\mathrm{HCN}(3-2)$ spectra at $265.89 \mathrm{GHz}$ : Solid line shows the best-fit spectrum, and dash line the spectrum calculated with the a priori HCN profile. Grey line is the observed data. The upper panel figure represents the zoom to the frequency range of $+/-100 \mathrm{MHz}$. Right: The retrieved HCN vertical profile (solid line) under the nominal temperature profile without considering the error in the flux calibration. The error bar indicates the 1- $\sigma$ limit of the retrieval. The dotted profile represents the a priori values where the gray shaded region corresponds to their errors. The ratio of the retrieved profile with respect to the a priori profile is also shown. On the extrem right panel, the averaging kernels of the retrieved $\mathrm{HCN}$ mixing ratios are shown.
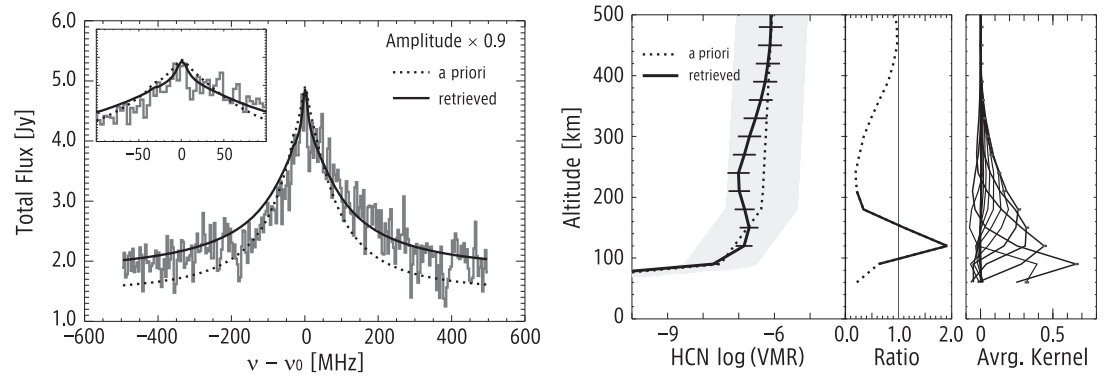

Fig. 4. See caption Fig. 3, but here the measurement data is scaled by a factor of 0.9 . 
Table 3. HCN mixing ratios in Titan's atmosphere

\begin{tabular}{ccccc}
\hline Altitude & Mixing ratio $[\mathrm{ppm}]$ & Wavelength $^{*}[\mu \mathrm{m}]$ & Facility/Instrument & Reference \\
\hline Stratosphere & $3.0 \times 10^{-7}$ & 3386.0 & IRAM-30m/SIS receiver & 38 \\
Stratosphere & $1.6 \times 10^{-7}$ & 14.025 & Voyager/IRIS & 39 \\
Stratosphere & $(0.75-52) \times 10^{-7}$ & 3386.0 & IRAM 30-m/SIS receiver & 40 \\
Stratosphere & $4.7 \times 10^{-8}-1.5 \times 10^{-6}$ & 14.025 & Voyager/ IRIS & 41 \\
$\quad 400 \mathrm{~km}$ & $\sim 2 \times 10^{-5}$ & & Model prediction & 42 \\
Stratosphere & $(0.5-4) \times 10^{-7}$ & 14.025 & IRAM 30-m/SIS receiver & 43 \\
$400 \mathrm{~km}$ & $1 \times 10^{-6}$ & & Model prediction & 44 \\
$700 \mathrm{~km}$ & $1 \times 10^{-5}$ & & Model prediction & 44 \\
$400 \mathrm{~km}$ & $1 \times 10^{-6}$ & 3386.0 & IRAM-30m/SIS receiver & 45 \\
$400 \mathrm{~km}$ & $1 \times 10^{-5}$ & & Model prediction & 46 \\
$700 \mathrm{~km}$ & $1 \times 10^{-4}$ & & Model prediction & 46 \\
Stratosphere & $3.0 \times 10^{-7}$ & 14.025 & ISO/SWS & 47 \\
$\sim 600 \mathrm{~km}$ & $7 \times 10^{-3}$ & 3 & Keck II/NIRSPEC & 46 \\
$83 \mathrm{~km}$ & $3 \times 10^{-5}$ & 1692.43 & SMA/5 and 6 antennas & 21 \\
$300 \mathrm{~km}$ & $3 \times 10^{-5}$ & 1692.43 & SMA/5 and 6 antennas & 21 \\
$500 \mathrm{~km}$ & $0.4 \times 10^{-6}$ & 14.04 & Cassini/CIRS & 48 \\
\hline
\end{tabular}

${ }^{*}$ In spite that each wavelength region is defined by standard specifical units, we use here $\mu \mathrm{m}$ to facilitate the comparisons.

\section{Discussion}

How does one explain the behavior of the retrieved HCN vertical profile obtained here? the relative poor signal-to-noise of the observations obtained here results in a poor fit to the HCN line wings. Furthermore, comparing the results obtained with previous results derived from disk-averaged information, interferometer measurements, and space-based observations summarized in Table 3 must be considered cautiously. In other words, direct comparison of the results from different instruments requires us to assume that all instrument-related offsets have been accounted for.

Certainly, Titan's atmospheric temperature and composition are not in a steady state. Does the HNC abundance retrieved here perhaps indicate that the composition of Titan's atmosphere has changed during the last years? Fig. 7 in Teanby et al. $2009^{5}$ shows that the HCN abundance measured by Cassini (at southern latitudes) has not significantly changed between 2006 and 2008. Then it seems to be that possible detections of time variations (if any) between different observations could be maybe due to observing more of the northern hemisphere than of the southern one as Titan's season changes. As we derived disk-averaged information we need to keep in mind, however, that our vertical distributions are mostly representative of the equatorial region of Titan since the measured flux density 


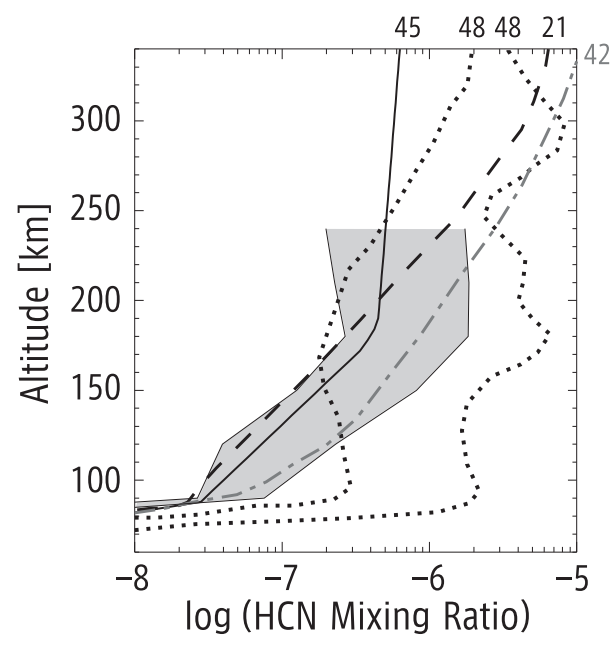

Fig. 5. Comparison of recent observational and modeling works of HCN abundances. The small numbers correspond to the indices of the reference on Table 3 . The shaded region represents the results here obtained, considering the 1- $\sigma$ limit of the retrieval error.

spectra are more heavily weighted toward equatorial latitudes. In case that vertical distributions would be representative of the northern latitudes, the profile analysis would become rather complicated. There is evidence of a vortex, which acts to separate enriched from unenriched air, at $25-55^{\circ} \mathrm{N}$ encircling the North Pole ${ }^{5,34,49}$.

Further higher signal-to-noise HCN observations at different times are necessary in order to open the possibility to detect seasonal and even spatial variations in $\mathrm{HCN}$, and confirm or not an enrichment layer in Titan's stratosphere.

\section{Summary}

- We report the first observations obtained with the SFHI APEX-1 instrument on APEX on a planetary/satellite atmosphere taken during SV. It consists of the spectra of $\mathrm{CO}(2-1)$ and $\mathrm{HCN}(3-2)$ in Titan's atmosphere.

- The observations reported here improved the control software of the APEX telescope, so that now it is possible to track planets with the APEX-1 receiver.

- We investigate the $\mathrm{CO}$ and HCN composition of Titan's stratosphere. Our CO mixing ratio estimation is consistent with other authors. We 
retrieved the HCN vertical profile, which is inconsistent with previous analyses. This requires further investigation by future $\mathrm{HCN}$ observations with higher signal-to-noise ratios. The search of nitriles and CO appears favorable in the sub-millimeter range explored with the APEX telescope and with the APEX-1 receiver.

This work represents a first step in exploring the capabilities of APEX and SHFI for Titan's atmospheric observations. New simultaneous observations of $\mathrm{HCN}$ and its isotopes at higher frequencies with APEX-2 and APEX-T2, for example, would improve the sensitivity to the abundance retrievals, and potentially could support, complement, and cross-calibrate the ESA's Herschel Space Observatory mission.

\section{Acknowledgments}

We are grateful to the APEX staff, in particular to Carlos De Breuck (ESO), for its assistance. We thank the anonymous reviewers for their constructive comments. This publication is based on data acquired with the Atacama Pathfinder Experiment (APEX). APEX is a collaboration between the Max-Planck-Institut für Radioastronomie, the European Southern Observatory, and the Onsala Space Observatory.

\section{References}

1. R. Hanel et al., Science 212, 192 (April 1981).

2. B. L. Lutz, C. de Bergh and T. Owen, Publ. Astron. Soc. Pac. 95, p. 593 (September 1983).

3. H. G. Roe, I. de Pater and C. P. McKay, Icarus 169, 440 (June 2004).

4. N. A. Teanby et al., Icarus 193, 595 (February 2008).

5. N. A. Teanby et al., Phil. Trans. R. Soc. A. 367, 697 (February 2009).

6. P. Hartogh et al., Planet. Space Sci. 57, 1596 (November 2009).

7. M. Rengel, H. Sagawa and P. Hartogh, Adv. Geo. in Press (2010).

8. R. Güsten et al., Astron. Astrophys. 454, L13 (August 2006).

9. E. Lellouch et al., Planet. Space Sci. 56, 1355 (August 2008).

10. V. Vassilev et al., Astron. Astrophys. 490, 1157 (November 2008).

11. O. Nyström et al., Journal of Infrared, Millimeter, and Terahertz Waves 30 , 746 (July 2009).

12. S. Vinatier, B. Bézard and C. A. Nixon, Icarus 191, 712 (November 2007).

13. E. de Vanssay et al., Planet. Space Sci. 43, 25 (February 1995).

14. P. Coll et al., Adv. Space Res. 16, 93 (August 1995).

15. A. Coupeaud et al., Laboratory study of the Titan's atmosphere chemistry: Formation and characterization of $\mathrm{HC}_{5} \mathrm{~N}$, in IAU Symposium, , IAU Symposium Vol. 235 (Cambridge University Press, California, August 2005).

16. M. Fulchignoni et al., Nature 438, 785 (December 2005). 
17. N. Semmoud-Monnanteuil and J. M. Colmont, J. Mol. Spec. 126, 210 (November 1987).

18. M. A. H. Smith et al., J. Mol. Spec. 105, 105 (May 1984).

19. R. Courtin, Icarus 75, 245 (August 1988).

20. A. Borysow and C. Tang, Icarus 105, 175 (September 1993).

21. M. A. Gurwell, Astrophys. J. Lett. 616, L7 (November 2004).

22. K. H. Baines et al., Planet. Space Sci. 54, 1552 (December 2006).

23. R. de Kok et al., Icarus 186, 354 (February 2007).

24. Y. L. Yung, M. Allen and J. P. Pinto, Astrophys. J. Suppl. 55, 465 (July 1984).

25. E. Lellouch et al., Icarus 162, 125 (March 2003).

26. M. A. López-Valverde, E. Lellouch and A. Coustenis, Icarus 175, 503 (June 2005).

27. T. Hidayat et al., Icarus 133, 109 (May 1998).

28. D. O. Muhleman, G. L. Berge and R. T. Clancy, Science 223, 393 (January 1984).

29. G. Paubert, D. Gautier and R. Courtin, Icarus 60, 599 (December 1984).

30. A. Marten et al., Icarus 76, 558 (December 1988).

31. M. A. Gurwell and D. O. Muhleman, Icarus 117, 375 (October 1995).

32. K. S. Noll et al., Icarus 124, 625 (December 1996).

33. M. A. Gurwell and D. O. Muhleman, Icarus 145, 653 (June 2000).

34. F. M. Flasar et al., Science 308, 975 (May 2005).

35. R. de Kok et al., Icarus 186, 354 (February 2007).

36. C. D. Rodgers, Rev. Geophys. Space Phys. 14, 609 (November 1976).

37. V. N. Markov et al., J. Mol. Spec. 212, 1 (March 2002).

38. G. Paubert et al., Bull. Am. Astron. Soc. 19, p. 633 (January 1987).

39. A. Coustenis, B. Bezard and D. Gautier, Icarus 80, 54 (July 1989).

40. L. Tanguy et al., Icarus 85, 43 (May 1990).

41. A. Coustenis and B. Bezard, Icarus 115, 126 (May 1995).

42. L. M. Lara et al., J. Geophys. Res. 101, 23261 (1996).

43. T. Hidayat et al., Icarus 126, 170 (March 1997).

44. S. Lebonnois et al., Icarus 152, 384 (August 2001).

45. A. Marten et al., Icarus 158, 532 (August 2002).

46. T. R. Geballe et al., Astrophys. J. Lett. 583, L39 (January 2003).

47. A. Coustenis et al., Icarus 161, 383 (February 2003).

48. N. A. Teanby et al., Icarus 186, 364 (February 2007).

49. R. K. Achterberg et al., Icarus 194, 263 (March 2008). 